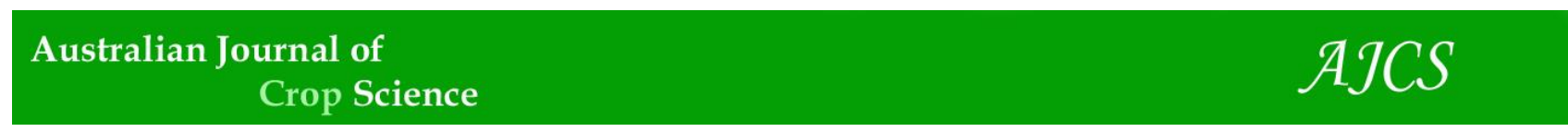

AJCS 13(05):773-779 (2019)

ISSN:1835-2707

doi: 10.21475/ajcs.19.13.05.p1565

\title{
Agronomic potential of biofortified crisphead lettuce (Lactuca sativa) and its reaction to Root- knot nematodes
}

\author{
Luciana Alves de Sousa ${ }^{1}$, Ana Carolina Pires Jacinto ${ }^{1}$, Aline José da Silveira ${ }^{1}$, Renata Castoldi ${ }^{1 *}$, Gabriel \\ Mascarenhas Maciel', Igor Forigo Beloti ${ }^{1}$
}

Instituto de Ciências Agrárias, Universidade Federal de Uberlândia, Monte Carmelo, MG, Brasil

\section{*Corresponding author: rcastoldi@ufu.br}

\begin{abstract}
Lettuce is the most-consumed leafy vegetable in Brazil due to its ease of preparation, nutritional quality, and affordability. Despite these benefits, there are few cultivars of crisphead lettuce (Lactuca sativa) that possess agronomic potential, high carotenoid content and resistance to nematodes. Therefore, we evaluated the agronomic potential of crisphead lettuce genotypes that are resistant to the root-knot nematode. The experiment was conducted at the Vegetable Experiment Station of the Federal University of Uberlandia (UFU), Monte Carmelo Campus. The trial was set up in a randomized block design with 49 treatments (43 genotypes obtained from crosses between Uberlandia 10000 and Belissima and six commercial cultivars) and three replications. After preparing the beds and before transplanting the seedlings, 11 simple samples were taken from each of the three experimental blocks to confirm the presence of nematodes within the experimental area. The following variables were determined 48 days after transplanting: chlorophyll content, leaf temperature, plant diameter, number of leaves per plant, fresh mass and incidence of root gall. The data were submitted to analysis of variance (ANOVA) and an F-test and the means were compared by the Scott-Knott test. Genetic diversity was represented by a dendrogram that was obtained using the hierarchical method of UPGMA and the optimization method of Tocher. We found genetic divergence and agronomic potential among the evaluated genotypes. Specifically, 199\#1\#1-E, 199\#2\#1-E, 199\#2\#2-E, 199\#3\#1-E, 199\#6\#1-E and 75\#2\#2-E presented good agronomic potential, were rich in carotenoids and showed resistance to the root-knot nematode.
\end{abstract}

Keywords: biofortification; genetic divergence; Lactuca sativa; Meloidogyne sp.; pro-vitamin A.

Abbreviations: SPAD_Soil Plant Analysis Development; IU_International Unit; N_nitrogen; Ca_calcium; Mg_magnesium; Na_sodium; K_potassium; H_hidrogen; Al_aluminum; P_phosphorus; SOM_Soil Organic Matter; pH_potential hydrogen; SB_Sum of Bases; CEC_Cation-exchange capacity.

\section{Introduction}

Lettuce is consumed worldwide without preparation and mainly in salads. In Brazil, it is the most consumed leafy vegetable and the third greatest in terms of production, trailing only watermelons and tomatoes (Abcsem, 2015).

Retail sales in Brazil reach approximately $\mathrm{R} \$ 8$ million reais per year while production surpasses 1.5 million tons (Sala and Costa 2012; Abcsem, 2015). In addition to its economic contribution through jobs and income generation, lettuce is also an important source of vitamins and minerals, especially vitamin A (as much as $4000 \mathrm{IU} / 100 \mathrm{~g}$ ) (Heredia Zárate et al., 2010). Vitamin A can be found as retinols and carotenoids (although only some carotenoids exist as provitamin A). Retinols are typically found in animal sources whereas carotenoids are found in plant-derived foods such as lettuce (Chapman, 2012; Penniston and Tanumihardjo, 2006). Vitamin A is important for vision, reproduction, embryogenic development, and immune function. Furthermore, carotenoid consumption has been associated with a lower risk of chronic diseases due to its antioxidant properties (Institute of Medicine, 2001).Concern in recent years about vitamin A deficiency, especially in less developed regions, has led to the production of cultivars with higher carotenoid content, which are an excellent low-cost alternative to vitamin A supplementation. One such cultivar is Uberlandia 10000 , which has more than 10,000 IUs of Vitamin A in $100 \mathrm{~g}$ of fresh leaves (equivalent to 36 millihenries $(\mathrm{mH})$ of $\beta$ carotene per $100 \mathrm{~g}$ of fresh leaves) (Sousa et al., 2007). Despite its nutritional advantages, the smooth-leaved Uberlandia 10000 has not gained popularity against curlyleaved and American type lettuce that currently lead the Brazilian market (Sala and Costa, 2012). Therefore, it is necessary to incorporate genes that control carotenoid content into different lettuce groups. It is also important to obtain cultivars that are resistant to certain pests and diseases, since, with intensified production, fields used for lettuce cultivation have been affected by phytopathogenic infestations. Currently, the root-knot nematode (specifically the Meloidogyne genus) is one of the main problems affecting lettuce cultivation. The pathogen can completely debilitate a plant by forming root galls that obstruct water and nutrient absorption from the soil. The high incidence of Meloidogyne sp. is attributed to its high reproductive 
capacity, especially in regions where soil temperatures are higher (Carvalho Filho et al., 2011a).

Resistant cultivars provide effective control without increasing production costs, except seed costs, and are compatible with other pest and disease maintenance methods (Ferreira et al., 2013). Therefore, we evaluated the agronomic potential of biofortified lettuce genotypes that are resistant to root-knot nematodes.

\section{Results}

\section{Agronomic evaluation}

All variables, except leaf temperature, had a significant effect ( $F$ Test, $5 \%$ probability) among the genotypes (Table 2).

The SPAD index, which indicates leaf chlorophyll content, showed that genotypes 199 \# 1-E, 199 \# 2-1-E, 199 \# 2-E, 199 \# 3 \# 1-E, 199 \# 6 \# 1-E and 75 \# 2 \# 2-E were significantly superior to the others, with, respectively, 209.29, 224.74, 223.42, 234.37, 209.15 and $226.54 \%$ more total chlorophyll than Uberlandia 10000 (which is considered rich in beta-carotene).

Leaf temperatures $\left({ }^{\circ} \mathrm{C}\right)$ ranged from 21.31 (189\#3\#4-E) to 28.02 (189\#3\#1-E) but did not vary significantly among treatments. Although there were no differences between the genotypes for this character, leaf temperatures were considered satisfactory.

The plant diameters $(\mathrm{cm})$ of the genotypes 117\#1\#3-E (39.46 $\mathrm{cm}), 75 \# 1 \# 1-\mathrm{E}(35.71 \mathrm{~cm})$ and 86\#2\#1-E $(36.92 \mathrm{~cm})$, were $142.61,129.06$ and $133.43 \%$ higher, respectively, than the Uberlandia 10000 cultivar.

The stem diameters of the largest genotypes ranged from $22.62 \mathrm{~mm}$ to $31.37 \mathrm{~mm}$ and were statistically equal to that of Uberlandia 10000. Therefore, these genotypes could be useful in advancing breeding programs for biofortified lettuce. These genotypes not only have high carotenoid levels, but also present agronomic characteristics that are similar or better than those of commercial cultivars.

The leaf numbers of $117 \# 1 \# 3-E, 184 \# 2 \# 5-E, 189 \# 2 \# 1-E$, 189\#3\#1-E, 190\#1\#2-E, 197\#1-E, 75\#1\#3-E did not differ significantly from Uberlandia 10000.

The highest fresh weights were found in $75 \# 1 \# 1-\mathrm{E}(0.36 \mathrm{~kg})$ and Grand Rapids Albina 1 (0.36 kg), which were 157.34 and $158.80 \%$ greater, respectively, than Uberlandia 10000.

\section{Root gall scores (SRNG)}

The genotypes 189\#3\#4-E; 189\#3\#2-E; 86\#1\#2-E; 120\#1\#1-E; 189\#3\#1-E; 107\#1\#1-E; 197\#1-E; 199\#2\#2-E; 189\#2\#2-E; 197\#2\#2-E; 199\#1\#1-E and 199\#3\#1-E were more resistant to the root-knot nematode (Meloidogyne sp.) than the susceptible Uberlandia 10000 cultivar and showed similar resistance to that of Grand Rapids (Table 3).

\section{Genetic dissimilarity}

The hierarchical UPGMA method (Figure 1) showed that the co-expressed correlation coefficient of the four groups was $87 \%$. Therefore, the matrix information and group formation were considered satisfactory.

The composition of these groups demonstrated wide genetic variability. The groups were separated by a $30 \%$ cut-off line that was established at points of abrupt change in the dendrogram (Cruz et al., 2012). These cut points yielded four distinct groups. Group I consisted of 21 genotypes, including the cultivar Belissima and the genotype Uberlandia 10000. Group II consisted of seven genotypes, group III, ten genotypes, including the Robusta cultivar, and finally group four consisted of eleven genotypes.

Mahalanobis $\mathrm{D}_{\mathrm{ii}}^{2}$ distance was used to measure dissimilarity, yielding four groups via the optimization method of Tocher (Table 4). Group I was made up of $91.84 \%$ of the genotypes, whereas groups II, III and IV were made up of only 4.08, 2.04 and $2.04 \%$, respectively.

The characteristics that differed most notably among the genotypes were the SPAD index and leaf temperature, as confirmed by the relative distribution of the characteristics ( 24.84 and $20.19 \%$ of total variability, respectively) (Table 5) while fresh weight contributed only $11.80 \%$.

\section{Discussion}

The genotypes under study can be considered rich in carotenoids since total chlorophyll and total carotenoids were highly correlated (Klooster et al., 2012). These genotypes could be viable options for breeding programs focused on carotenoid-rich lettuce.

Moderately high leaf temperatures (35 to $42{ }^{\circ} \mathrm{C}$ ) can negatively affect photosynthesis, causing changes in the thylakoid membrane and altering physicochemical properties (Dias and Marenco, 2007), which in turn may interfere with chlorophyll content and inflate the SPAD index.

The genotypes $117 \# 1 \# 3-E$, 75\#1\#1-E and $86 \# 2 \# 1-E$ could be excellent options for wholesalers and retailers given that consumers value larger heads of lettuce. Conversely, larger plants may be susceptible to damage during packaging and transport (Suinaga et al., 2013), which could reduce commercial quality.

The plant diameter $(21.4$ to $25.6 \mathrm{~cm}$ ) of loose-leaf lettuce grown under high temperatures (Santos et al., 2009) was lower than that of the genotypes in the present experiment ( 35.71 to $39.46 \mathrm{~cm}$ ). This discrepancy could be explained by temperature differences between growing regions and the sensitivity of lettuce to adverse temperatures, humidity, and rainfall (Ferreira et al., 2010).

According to Oliveira et al. (2004), leaf number is the most important component of plant development. This is significant for lettuce given that this metric is a commercial priority. Leaf numbers in the current study were much higher than those found by Aquino et al. (2017), which ranged from 12.8 to 15.6 in loose-leaf lettuce cultivars. According to Sala and Costa (2012), sale of processed and packaged lettuce is growing in Brazil, the United States and Europe. Thus, higher leaf numbers would help meet this growing market demand.

Air temperature affects plant development, leaf emissions and growth and is the most important environmental factor for many crops (Hermes et al., 2001). Given their relative newness, the genotypes in the current study should be evaluated in different regions, seasons and environments to better understand their climatic adaptations and to select those with the highest yields.

The fresh weights recorded in the present study agree with Feltrin et al., (2009), who found values from 210.68 to $338.99 \mathrm{~g}$ for winter and summer crops of field-grown and hydroponic crisphead lettuce. Feltrin et al., (2009) also 
Table 1. Genotypes of crisphead lettuce (Lactuca sativa).

\begin{tabular}{|c|c|}
\hline Treatments & Genotypes \\
\hline 1 & Belíssima \\
\hline 2 & $107 \# 1 \# 1-\mathrm{E}$ \\
\hline 3 & $107 \# 1 \# 2-E$ \\
\hline 4 & $117 \# 1 \# 1-\mathrm{E}$ \\
\hline 5 & $117 \# 1 \# 3-E$ \\
\hline 6 & 120\#1\#1-E \\
\hline 7 & $125 \# 1 \# 1-E$ \\
\hline 8 & $125 \# 2 \# 2-E$ \\
\hline 9 & $184 \# 2$ \\
\hline 10 & $184 \# 2 \# 1-E$ \\
\hline 11 & $184 \# 2 \# 5-E$ \\
\hline 12 & $189 \# 1 \# 2-E$ \\
\hline 13 & $189 \# 2 \# 1-\mathrm{E}$ \\
\hline 14 & $189 \# 2 \# 2-E$ \\
\hline 15 & $189 \# 2 \# 3-E$ \\
\hline 16 & 189\#3\#1-E \\
\hline 17 & $189 \# 3 \# 2-\mathrm{E}$ \\
\hline 18 & 189\#3\#3-E \\
\hline 19 & $189 \# 3 \# 4-E$ \\
\hline 20 & $190 \# 1 \# 2-E$ \\
\hline 21 & $197 \# 1-\mathrm{E}$ \\
\hline 22 & $197 \# 2 \# 1-\mathrm{E}$ \\
\hline 23 & $197 \# 2 \# 2-E$ \\
\hline 24 & $197 \# 3 \# 1-\mathrm{E}$ \\
\hline 25 & $199 \# 1 \# 1-\mathrm{E}$ \\
\hline 26 & $199 \# 2 \# 1-E$ \\
\hline 27 & $199 \# 2 \# 2-\mathrm{E}$ \\
\hline 28 & $199 \# 3 \# 1-E$ \\
\hline 29 & $199 \# 5 \# 2-\mathrm{E}$ \\
\hline 30 & $199 \# 6 \# 1-\mathrm{E}$ \\
\hline 31 & $206 \# 1 \# 2-E$ \\
\hline 32 & $206 \# 1 \# 4-E$ \\
\hline 33 & 206\#1\#6-E \\
\hline 34 & 206\#3\#1-E \\
\hline 35 & $7 \# 2 \# 1-E$ \\
\hline 36 & 7\#2\#2-E \\
\hline 37 & 75\#1\#1-E \\
\hline 38 & $75 \# 1 \# 2-E$ \\
\hline 39 & $75 \# 1 \# 3-E$ \\
\hline 40 & $75 \# 2 \# 2-E$ \\
\hline 41 & 75\#3\#1-E \\
\hline 42 & $75 \# 3 \# 2-E$ \\
\hline 43 & $86 \# 1 \# 2-E$ \\
\hline 44 & $86 \# 2 \# 1-E$ \\
\hline 45 & Grand Rapids Albina \#1 \\
\hline 46 & Grand Rapids Albina \#2 \\
\hline 47 & Grand Rapids Albina \#3 \\
\hline 48 & Robusta \\
\hline 49 & Uberlândia 10000 \\
\hline
\end{tabular}

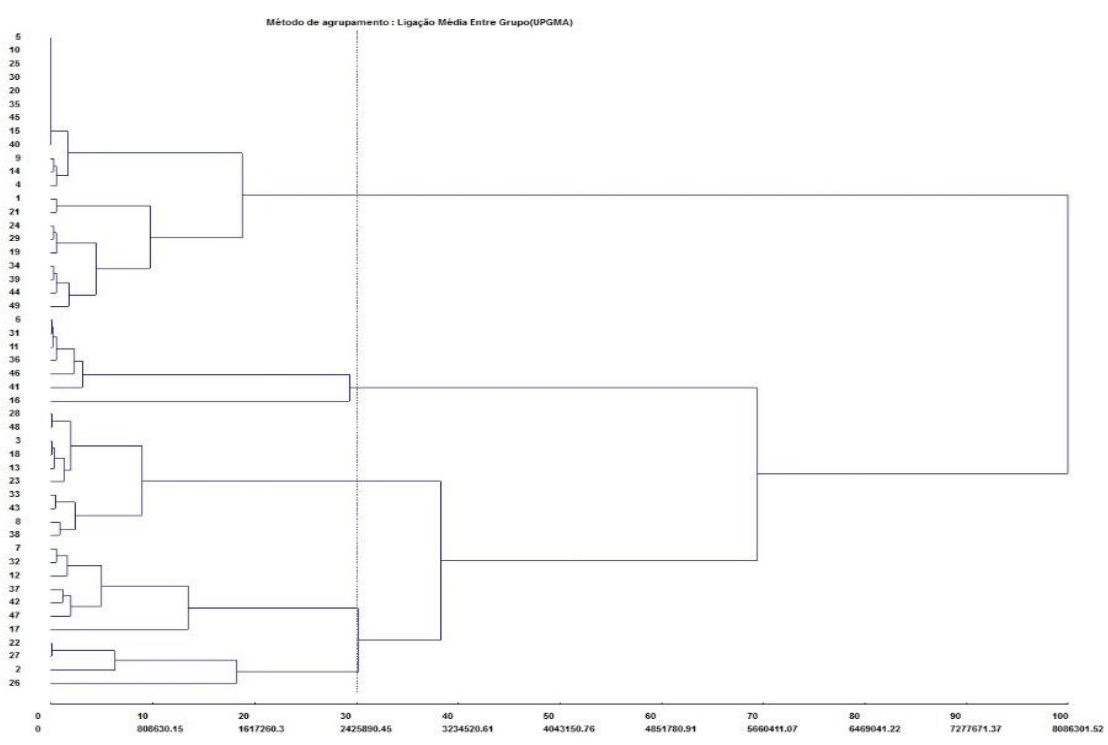

Fig 1. Dendrogram of genetic divergence among 49 lettuce lines, obtained by the UPGMA hierarchical method as a measure of dissimilarity. The genotypes code can be visualized according to Table 1 . 
Table 2. Agronomic evaluation of biofortified lettuce SPAD, leaf temperature (LT), plant diameter (PD), stem diameter (SD), number of leaves (NF) and fresh weight (FW).

\begin{tabular}{|c|c|c|c|c|c|c|}
\hline Genotype & SPAD & $\mathrm{LT}\left({ }^{\circ} \mathrm{C}\right)$ & $\mathrm{PD}(\mathrm{cm})$ & $\mathrm{SD}(\mathrm{mm})$ & $\mathrm{NF}$ & FW (kg) \\
\hline Belíssima & $13.44 D^{*}$ & $25.84 \mathrm{~A}$ & $14.26 \mathrm{D}$ & $11.91 \mathrm{C}$ & $14.83 \mathrm{D}$ & $0,05 \mathrm{G}$ \\
\hline 107\#1\#1-E & $22.14 \mathrm{C}$ & $23,84 \mathrm{~A}$ & $26.63 \mathrm{C}$ & $24.79 \mathrm{~A}$ & 30.83 B & $0.10 \mathrm{~F}$ \\
\hline $107 \# 1 \# 2-E$ & 24.98 B & $24.21 \mathrm{~A}$ & $24.34 \mathrm{C}$ & $23.22 \mathrm{~A}$ & 29.17 B & $0.12 \mathrm{E}$ \\
\hline $117 \# 1 \# 1-E$ & $14.89 \mathrm{D}$ & $23.86 \mathrm{~A}$ & 28.33 B & $29.76 \mathrm{~A}$ & 32.83 B & $0.24 \mathrm{C}$ \\
\hline $117 \# 1 \# 3-E$ & $15.70 \mathrm{D}$ & $23.87 \mathrm{~A}$ & $39.46 \mathrm{~A}$ & $31.37 \mathrm{~A}$ & $42.08 \mathrm{~A}$ & 0.31 B \\
\hline $120 \# 1 \# 1-E$ & $13.61 \mathrm{D}$ & $23.81 \mathrm{~A}$ & $19.84 \mathrm{D}$ & $16.55 \mathrm{C}$ & 17.17 D & $0.15 \mathrm{E}$ \\
\hline $125 \# 1 \# 1-\mathrm{E}$ & $15.56 \mathrm{D}$ & $23.56 \mathrm{~A}$ & $25.54 \mathrm{C}$ & $26.89 \mathrm{~A}$ & $23.17 \mathrm{C}$ & $0.11 \mathrm{~F}$ \\
\hline $125 \# 2 \# 2-\mathrm{E}$ & $18.90 \mathrm{C}$ & $22.79 \mathrm{~A}$ & $23.25 \mathrm{C}$ & $25.79 \mathrm{~A}$ & $27.17 \mathrm{C}$ & $0.17 \mathrm{D}$ \\
\hline $184 \# 2$ & $27.39 \mathrm{~B}$ & $22.39 \mathrm{~A}$ & 30.71 B & $25.59 \mathrm{~A}$ & $31.00 \mathrm{~B}$ & $0.20 \mathrm{D}$ \\
\hline $184 \# 2 \# 1-E$ & $25.89 \mathrm{~B}$ & $24.67 \mathrm{~A}$ & 27.75 B & $22.62 \mathrm{~A}$ & 30.25 B & $0.17 \mathrm{D}$ \\
\hline $184 \# 2 \# 5-E$ & 25.02 B & $23.89 \mathrm{~A}$ & $32.00 \mathrm{~B}$ & $27.95 \mathrm{~A}$ & $36.58 \mathrm{~A}$ & $0.25 \mathrm{C}$ \\
\hline $189 \# 1 \# 2-\mathrm{E}$ & $19.85 \mathrm{C}$ & $25.17 \mathrm{~A}$ & $23.67 \mathrm{C}$ & $20.60 \mathrm{~B}$ & 31.08 B & $0.18 \mathrm{D}$ \\
\hline $189 \# 2 \# 1-E$ & $19.60 \mathrm{C}$ & $21.84 \mathrm{~A}$ & $25.54 \mathrm{C}$ & $23.24 \mathrm{~A}$ & $34.17 \mathrm{~A}$ & $0.16 \mathrm{D}$ \\
\hline $189 \# 2 \# 2-E$ & $23.20 \mathrm{C}$ & $23.25 \mathrm{~A}$ & $24.13 \mathrm{C}$ & $22.75 \mathrm{~A}$ & 28.92 B & $0.15 \mathrm{E}$ \\
\hline $189 \# 2 \# 3-E$ & $21.99 \mathrm{C}$ & $26.01 \mathrm{~A}$ & $26.21 \mathrm{C}$ & $22.69 \mathrm{~A}$ & 31.16 B & $0.17 \mathrm{D}$ \\
\hline 189\#3\#1-E & $16.18 \mathrm{D}$ & $28.02 \mathrm{~A}$ & $19.92 \mathrm{D}$ & $22.74 \mathrm{~A}$ & $34.75 \mathrm{~A}$ & $0.13 \mathrm{E}$ \\
\hline $189 \# 3 \# 2-\mathrm{E}$ & 17.14 D & $22.15 \mathrm{~A}$ & $24.50 \mathrm{C}$ & $26.31 \mathrm{~A}$ & 30.25 B & $0.14 \mathrm{E}$ \\
\hline $189 \# 3 \# 3-E$ & 17.12 D & $24.61 \mathrm{~A}$ & $23.63 \mathrm{C}$ & $23.25 \mathrm{~A}$ & 30.67 B & $0.13 \mathrm{E}$ \\
\hline $189 \# 3 \# 4-E$ & 24.29 B & $21.31 \mathrm{~A}$ & $18.00 \mathrm{D}$ & $17.38 \mathrm{C}$ & $25.92 \mathrm{C}$ & $0.07 \mathrm{G}$ \\
\hline $190 \# 1 \# 2-E$ & $22.22 \mathrm{C}$ & $24.74 \mathrm{~A}$ & 29.88 B & $24.45 \mathrm{~A}$ & $37.5 \mathrm{~A}$ & $0.24 \mathrm{C}$ \\
\hline $197 \# 1-E$ & $23.13 \mathrm{C}$ & $23.44 \mathrm{~A}$ & $27.17 \mathrm{C}$ & $26.37 \underline{a}$ & $34.25 \mathrm{~A}$ & $0.21 \mathrm{D}$ \\
\hline $197 \# 2 \# 1-E$ & $18.25 \mathrm{D}$ & $24.08 \mathrm{~A}$ & $23.42 \mathrm{C}$ & $21.90 \mathrm{~B}$ & $27.42 \mathrm{C}$ & $0.15 \mathrm{E}$ \\
\hline $197 \# 2 \# 2-E$ & $20.11 \mathrm{C}$ & $24.40 \mathrm{~A}$ & $25.71 \mathrm{C}$ & $23.57 \mathrm{~A}$ & $25.58 \mathrm{C}$ & $0.18 \mathrm{D}$ \\
\hline $197 \# 3 \# 1-E$ & $22.83 \mathrm{C}$ & $23.77 \mathrm{~A}$ & $25.50 \mathrm{C}$ & $25.57 \mathrm{~A}$ & $22.92 \mathrm{C}$ & $0.20 \mathrm{D}$ \\
\hline 199\#1\#1-E & $30.20 \mathrm{~A}$ & $22.31 \mathrm{~A}$ & $25.46 \mathrm{C}$ & 21.71 B & $26.33 \mathrm{C}$ & $0.19 \mathrm{D}$ \\
\hline $199 \# 2 \# 1-E$ & $32.43 \mathrm{~A}$ & $22.74 \mathrm{~A}$ & $31.88 \mathrm{~B}$ & 19.38 B & $28.08 \mathrm{C}$ & $0.18 \mathrm{D}$ \\
\hline $199 \# 2 \# 2-E$ & $32.24 \mathrm{~A}$ & $27.38 \mathrm{~A}$ & $31.54 \mathrm{~B}$ & 20.33 B & $25.17 \mathrm{C}$ & $0.17 \mathrm{D}$ \\
\hline 199\#3\#1-E & $33.82 \mathrm{~A}$ & $24.08 \mathrm{~A}$ & 29.25 B & 20.75 B & 30.58 B & $0.13 \mathrm{E}$ \\
\hline $199 \# 5 \# 2-E$ & $17.91 \mathrm{D}$ & $21.78 \mathrm{~A}$ & $23.13 \mathrm{C}$ & $22.21 \mathrm{~B}$ & $24.33 \mathrm{C}$ & $0.17 \mathrm{D}$ \\
\hline 199\#6\#1-E & $30.18 \mathrm{~A}$ & $24.92 \mathrm{~A}$ & $30.25 \mathrm{~B}$ & 21.73 B & 30.67 B & $0.20 \mathrm{D}$ \\
\hline $206 \# 1 \# 2-E$ & $19.95 \mathrm{C}$ & $21.54 \mathrm{~A}$ & $24.21 \mathrm{C}$ & $25.17 \mathrm{~A}$ & $22.75 \mathrm{C}$ & $0.19 \mathrm{D}$ \\
\hline 206\#1\#4-E & $17.50 \mathrm{D}$ & $21.86 \mathrm{~A}$ & $22.34 \mathrm{C}$ & $23.56 \mathrm{~A}$ & $20.83 \mathrm{D}$ & $0.15 \mathrm{E}$ \\
\hline 206\#1\#6-E & $21.27 \mathrm{C}$ & $25.14 \mathrm{~A}$ & $21.21 \mathrm{D}$ & 21.61 B & $19.58 \mathrm{D}$ & $0.23 \mathrm{C}$ \\
\hline 206\#3\#1-E & $21.09 \mathrm{C}$ & $24.64 \mathrm{~A}$ & 29.92 B & $29.87 \mathrm{~A}$ & $27.33 \mathrm{C}$ & $0.26 \mathrm{C}$ \\
\hline 7\#2\#1-E & $21.73 \mathrm{C}$ & $25.78 \mathrm{~A}$ & $26.08 \mathrm{C}$ & $25.16 \mathrm{~A}$ & $24.75 \mathrm{C}$ & $0.14 \mathrm{E}$ \\
\hline 7\#2\#2-E & $19.61 \mathrm{C}$ & $21.70 \mathrm{~A}$ & 29.96 B & $27.09 \mathrm{~A}$ & $27.58 \mathrm{C}$ & $0.21 \mathrm{C}$ \\
\hline 75\#1\#1-E & 17.07 D & $24.66 \mathrm{~A}$ & $35.71 \mathrm{~A}$ & $24.92 \mathrm{~A}$ & $31.50 \mathrm{~B}$ & $0.36 \mathrm{~A}$ \\
\hline $75 \# 1 \# 2-E$ & 24.18 B & $21.49 \mathrm{~A}$ & $26.17 \mathrm{C}$ & 22.14 B & 29.00 B & $0.14 \mathrm{E}$ \\
\hline 75\#1\#3-E & $22.50 \mathrm{C}$ & $24.31 \mathrm{~A}$ & $29.40 \mathrm{~B}$ & $25.78 \mathrm{~A}$ & $38.50 \mathrm{~A}$ & $0.18 \mathrm{D}$ \\
\hline 75\#2\#2-E & $32.69 \mathrm{~A}$ & $23.94 \mathrm{~A}$ & 32.08 B & 19.15 B & $24.92 \mathrm{C}$ & $0.18 \mathrm{D}$ \\
\hline 75\#3\#1-E & $20.62 \mathrm{C}$ & $24.99 \mathrm{~A}$ & $32.46 \mathrm{~B}$ & $23.16 \mathrm{~A}$ & $27.25 \mathrm{C}$ & $0.26 \mathrm{C}$ \\
\hline $75 \# 3 \# 2-E$ & $20.04 \mathrm{C}$ & $23.37 \mathrm{~A}$ & 29.63 B & 19.27 B & $25.25 \mathrm{C}$ & $0.17 \mathrm{D}$ \\
\hline $86 \# 1 \# 2-E$ & 16.29 D & $26.42 \mathrm{~A}$ & 29.17 B & 20.48 B & $26.75 \mathrm{C}$ & $0.16 \mathrm{D}$ \\
\hline $86 \# 2 \# 1-E$ & $17.73 \mathrm{D}$ & $24.76 \mathrm{~A}$ & $36.92 \mathrm{~A}$ & 21.63 B & 29.42 B & $0.25 \mathrm{C}$ \\
\hline Grand Rapids Albina \#1 & 24.06 B & $25.04 \mathrm{~A}$ & 29.75 B & $19.70 \mathrm{~B}$ & $26.00 \mathrm{C}$ & $0.36 \mathrm{~A}$ \\
\hline Grand Rapids Albina \#2 & $19.69 \mathrm{C}$ & $25.76 \mathrm{~A}$ & $25.88 \mathrm{C}$ & $14.04 \mathrm{C}$ & $17.42 \mathrm{D}$ & $0.19 \mathrm{D}$ \\
\hline Grand Rapids Albina \#3 & 17.15 D & $21.30 \mathrm{~A}$ & $27.46 \mathrm{~B}$ & $17.46 \mathrm{C}$ & $21.75 \mathrm{D}$ & $0.24 \mathrm{C}$ \\
\hline Robusta & $14.35 \mathrm{D}$ & $23.94 \mathrm{~A}$ & $23.50 \mathrm{C}$ & 20.51 B & $20.5 \mathrm{D}$ & $0.14 \mathrm{E}$ \\
\hline Uberlândia 10000 & $14.43 \mathrm{D}$ & $25.14 \mathrm{~A}$ & $27.67 \mathrm{~B}$ & $25.70 \mathrm{~A}$ & $34.58 \mathrm{~A}$ & $0.23 \mathrm{C}$ \\
\hline
\end{tabular}

*Means followed by distinct letters within columns differ statistically by the Scott-Knott test at 0.05 .

Table 3. The reaction of lettuce to nematodes.

\begin{tabular}{llll} 
Table 3. The reaction of lettuce to nematodes. & & SRNG (\%) & Standard deviation \\
\hline Genotype & Averages & $-88.03^{* *}$ & 0.33 \\
\hline Robusta & $0.17 \mathrm{~A}$ & -76.76 & 0.00 \\
Grand Rapids Albina \#2 & $0.33 \mathrm{~A}$ & -70.42 & 0.17 \\
Grand Rapids Albina \#1 & $0.42 \mathrm{~A}$ & -64.79 & 0.33 \\
189\#3\#4-E & $0.50 \mathrm{~A}$ & -59.15 & 0.36 \\
189\#3\#2-E & $0.58 \mathrm{~A}$ & -52.82 & 0.33 \\
$86 \# 1 \# 2-E$ & $0.67 \mathrm{~A}$ & -52.82 & 0.68 \\
120\#1\#1-E & $0.67 \mathrm{~A}$ & -47.18 & 0.63 \\
Grand Rapids Albina \#3 & $0.75 \mathrm{~A}$ & -38.03 & 0.15 \\
189\#3\#1-E & $0.88 \mathrm{~A}$ & -35.21 & 0.32 \\
107\#1\#1-E & $0.92 \mathrm{~A}$ & -29.58 & 0.76 \\
197\#1-E & $1.00 \mathrm{~A}$ & -17.61 & 0.49 \\
199\#2\#2-E & $1.17 \mathrm{~A}$ & -17.61 & 0.86 \\
189\#2\#2-E & $1.17 \mathrm{~A}$ & -11.97 & 0.51 \\
197\#2\#2-E & $1.25 \mathrm{~A}$ &
\end{tabular}




\begin{tabular}{|c|c|c|c|}
\hline 199\#1\#1-E & $1.33 \mathrm{~A}$ & -6.34 & 0.67 \\
\hline 199\#3\#1-E & $1.33 \mathrm{~A}$ & -6.34 & 0.49 \\
\hline Uberlândia 10000 & $1.42 \mathrm{~A}$ & 0.00 & 0.76 \\
\hline 75\#1\#1-E & $1.42 \mathrm{~A}$ & 0.00 & 0.80 \\
\hline 199\#6\#1-E & $1.42 \mathrm{~A}$ & 0.00 & 0.44 \\
\hline $189 \# 2 \# 1-E$ & $1.42 \mathrm{~A}$ & 0.00 & 0.64 \\
\hline $197 \# 2 \# 1-E$ & $1.42 \mathrm{~A}$ & 0.00 & 0.59 \\
\hline 199\#2\#1-E & $1.42 \mathrm{~A}$ & 0.00 & 0.44 \\
\hline $7 \# 2 \# 2-E$ & $1.42 \mathrm{~A}$ & 0.00 & 0.65 \\
\hline $75 \# 3 \# 2-E$ & $1.42 \mathrm{~A}$ & 0.00 & 0.42 \\
\hline $75 \# 2 \# 2-E$ & $1.50 \mathrm{~A}$ & 5.63 & 0.68 \\
\hline 197\#3\#1-E & $1.58 \mathrm{~A}$ & 11.27 & 1.00 \\
\hline Belíssima & $1.58 \mathrm{~A}$ & 11.27 & 0.69 \\
\hline $184 \# 2 \# 5-E$ & $1.67 \mathrm{~A}$ & 17.61 & 0.80 \\
\hline 86\#2\#1-E & $1.67 \mathrm{~A}$ & 17.61 & 0.61 \\
\hline $184 \# 2 \# 1-E$ & $1.75 \mathrm{~B}$ & 23.24 & 0.36 \\
\hline 125\#1\#1-E & $1.83 \mathrm{~B}$ & 28.87 & 0.33 \\
\hline $107 \# 1 \# 2-E$ & $1.83 \mathrm{~B}$ & 28.87 & 0.67 \\
\hline $125 \# 2 \# 2-E$ & $1.92 \mathrm{~B}$ & 35.21 & 1.13 \\
\hline 206\#1\#4-E & $1.92 \mathrm{~B}$ & 35.21 & 0.79 \\
\hline $184 \# 2$ & $2.00 \mathrm{~B}$ & 40.85 & 0.76 \\
\hline 189\#1\#2-E & $2.08 \mathrm{~B}$ & 46.48 & 0.59 \\
\hline $206 \# 1 \# 2-E$ & $2.08 \mathrm{~B}$ & 46.48 & 1.09 \\
\hline 117\#1\#1-E & $2.25 \mathrm{~B}$ & 58.45 & 0.94 \\
\hline 117\#1\#3-E & $2.33 \mathrm{~B}$ & 64.08 & 0.94 \\
\hline 75\#3\#1-E & $2.33 \mathrm{~B}$ & 64.08 & 1.15 \\
\hline 75\#1\#2-E & $2.42 \mathrm{~B}$ & 70.42 & 0.36 \\
\hline $199 \# 5 \# 2-E$ & $2.50 \mathrm{~B}$ & 76.06 & 0.67 \\
\hline 206\#1\#6-E & $2.58 \mathrm{~B}$ & 81.69 & 0.50 \\
\hline 75\#1\#3-E & $2.58 \mathrm{~B}$ & 81.69 & 0.50 \\
\hline 189\#3\#3-E & $2.67 \mathrm{~B}$ & 88.03 & 1.21 \\
\hline $190 \# 1 \# 2-\mathrm{E}$ & $2.75 \mathrm{~B}$ & 93.66 & 0.82 \\
\hline 206\#3\#1-E & $2.75 \mathrm{~B}$ & 93.66 & 1.02 \\
\hline 7\#2\#1-E & $2.92 \mathrm{~B}$ & i5.63 & 1.34 \\
\hline $189 \# 2 \# 3-E$ & $3.58 \mathrm{~B}$ & 152.11 & 0.84 \\
\hline
\end{tabular}

Table 4. Grouping of 49 lettuce lines by the Tocher method.

\begin{tabular}{|c|c|}
\hline Group & Members \\
\hline \multirow{3}{*}{1} & $\begin{array}{l}\text { 199\#2\#1-E; 75\#2\#2-E;199\#3\#1-E;199\#1\#1-E;199\#6\#1-E;184\#2\#1-E;184\#2;189\#2\#2-E;75\#1\#2-E; } 197 \# 2 \# 2- \\
\mathrm{E} ; 197 \# 3 \# 1-\mathrm{E} ; 197 \# 2 \# 1-\mathrm{E} ; 189 \# 2 \# 3-\mathrm{E} ; 107 \# 1 \# 2-\mathrm{E} ; 7 \# 2 \# 1-\mathrm{E} ; 107 \# 1 \# 1-\mathrm{E} ; 125 \# 2 \# 2-\mathrm{E} ; 189 \# 1 \# 2-\mathrm{E} ; 189 \# 3 \# 3-\mathrm{E} ; 75 \# 3 \# 2-\end{array}$ \\
\hline & E;197\#1-E;189\#2\#1-E;199\#5\#2-E;189\#3\#2-E;206\#1\#2-E;206\#1\#4-E;7\#2\#2-E;75\#1\#3-E;Crespa \\
\hline & $\begin{array}{l}\text { Robusta;86\#1\#2-E;206\#1\#6-E;75\#3\#1-E;190\#1\#2-E;Uberlândia 10000;117\#1\#1-E;184\#2\#5-E;206\#3\#1-E;86\#2\#1- } \\
\text { E;199\#2\#2-E; Grand Rapids Albina \#3;120\#1\#1-E;189\#3\#1-E;189\#3\#4-E; Grand Rapids Albina \#2; }\end{array}$ \\
\hline II & 117\#1\#3-E;75\#1\#1-E \\
\hline III & Grand Rapids Albina \#1 \\
\hline IV & Belíssima \\
\hline
\end{tabular}

Table 5. Relative contribution of six characteristics to the genetic divergence of 49 genotypes of biofortified lettuce, according to the criteria of Singh (1981).

\begin{tabular}{lll}
\hline Characteristics & $\mathrm{S} . \mathrm{j}$ & $\mathrm{S} . \mathrm{j}(\%)$ \\
\hline SPAD & 156.24 & 24.84 \\
Leaf temperature $(\stackrel{\circ}{ } \mathrm{C})$ & 127.01 & 20.19 \\
Plant diameter $(\mathrm{cm})$ & 81.59 & 12.97 \\
Stem diameter $(\mathrm{mm})$ & 90.12 & 14.32 \\
Number of leaves & 99.88 & 15.88 \\
Fresh weight $(\mathrm{Kg})$ & 74.23 & 11.80 \\
\hline
\end{tabular}

found that the fresh weight of winter lettuce $(346.97 \mathrm{~g})$ was always greater than that of summer lettuce $(247.15 \mathrm{~g})$, regardless of cultivation system (hydroponic or in the field). Even though the current experiment was conducted under high summer temperatures (February to April in the southern hemisphere), some of the genotypes yielded fresh weight values were similar or higher than those reported by Feltrin et al. (2009).

Several authors (Carvalho Filho et al., 2011a; Ferreira et al., 2011) have reported the resistance of the Grand Rapids cultivar to root-knot nematodes. While gall incidence may not be the most efficient way to demonstrate nematode resistance or susceptibility, it is still be the best option for selecting genotypes in breeding programs because it is practical and does not destroy the plants (Carvalho Filho et al., 2011b).

Although the number of groups found by the optimization method of Tocher and the hierarchical method of UPGMA were equal (four groups), there were noticeable differences in the number of genotypes per group. Similar disagreement 
between multivariate methods was also observed by Nunes et al. (2011), who worked with other species.

Nick et al. (2010) stated the importance of identifying characteristics that most contribute to genetic diversity and those that could be discarded due to their insignificance.

\section{Materials and Methods}

\section{Plant material and location of the experiment}

The experiment was conducted between February and April, 2017, at the Vegetable Experiment Station of the Federal University of Uberlandia, Monte Carmelo Campus (873 m above sea level, 1842'43.19 "S, 4729'55.8"W). The treatments consisted of 43 genotypes from the fifth selffertilization of a cross between the Uberlandia 10000 and Belissima cultivars and six commercial cultivars (Belissima, Uberlandia 10000, Robusta, Grand Rapids Albina 1, Grand Rapids Albina 2 and Grand Rapids Albina 3) (Table 1).

\section{Experimental design and treatments}

A randomized-block experimental design was used with 49 treatments (Table 1) and three replications. The following statistical model was used: $Y i j=\mu+b j+t i+e i j$, where: $Y i j$ is the observation of the $\mathrm{i}$-th genotype in the $\mathrm{j}$-th block; $\mu$ is the fixed effect of the global average; gi is the effect of the ith genotype; bj: the effect of the j-th block; and eij the mean experimental error. Each plot consisted of 16 plants, where only the four centermost plants were considered.

\section{Procedures}

Seeds were sowed in 200-cell polystyrene trays that had been filled with a commercially available substrate of coconut fiber. After sowing, the trays were maintained in a hoop-style greenhouse (area: $5 \times 6 \mathrm{~m}$, height: $3.5 \mathrm{~m}$ ) that was covered with UV resistant polyethylene film (150 microns) and anti-aphid side screens.

Forty-four days after sowing, the seedlings were transplanted to beds in the field that had been prepared and fertilized according to soil analysis and crop recommendations.

Before setting up the experiment, soil samples were taken from a depth of $0-20 \mathrm{~cm}$ and analyzed at the Soil Fertility Laboratory of the Federal University of Uberlandia. The physical/chemical analysis showed the following: clayey texture (>50\%); $\mathrm{pH}$ in $\mathrm{CaCl}_{2}=4.9$; $\mathrm{SOM}=3.9 \mathrm{dag} \mathrm{kg}^{-1}$; $\mathrm{P}(\mathrm{rem})=79.1 \mathrm{mg} \mathrm{dm}^{-3} ; \mathrm{K}=0.29 \mathrm{cmol}^{-\mathrm{dm}^{-3}} ; \mathrm{Ca}=3.3 \mathrm{cmolc}$ $\mathrm{dm}^{-3} ; \mathrm{Mg}=1.3 \mathrm{cmolcdm}^{-3} ; \mathrm{H}+\mathrm{Al}=4.9 \mathrm{cmolcdm}^{-3} ; \mathrm{SB}=4.90$ $\mathrm{cmolcdm}^{-3} ; \mathrm{CEC}=9.80 \mathrm{cmolcdm}^{-3} ; \mathrm{BS} \%=50$.

The soil analysis, crop requirements and recommendations of Ribeiro et al. (1999) were used to calculate the fertilizers needed for planting, broadcasting, and liming. The experiment was conducted in a naturally infected soil. After soil preparation and before transplanting, eleven simple samples were collected $(0-25 \mathrm{~cm}$ depth) from each of the three blocks to determine the existence, genus types, and quantity of nematodes present. The nematode analysis (Jenkins, 1964) showed the incidence of Meloidogyne sp. with 174.9 adults per $100 \mathrm{~cm}^{3}$ of soil, 54.0 adults per 100 $\mathrm{cm}^{3}$ of soil and 92.25 adults per $100 \mathrm{~cm}^{3}$ of soil in blocks I, II and III, respectively.
The plants were irrigated by sprinklers (spaced $12.0 \times 12.0$ $\mathrm{m})$ with an individual flow rate of $0.45 \mathrm{~m}^{3} \mathrm{~h}^{-1}$.

\section{Variables}

The following evaluations were carried out 48 days after transplanting: a) chlorophyll content: measured in the morning from a central leaf using a SPAD chlorophyll meter (Minolta SPAD-502, Konica Minolta, Nova Jersey, USA); b) leaf temperature $\left({ }^{\circ} \mathrm{C}\right)$ using an infrared thermometer $(300-$ EN-01, Quick-Temp, China) that was held at a constant distance from the leaf; c) plant diameter $(\mathrm{cm})$ using a ruler; d) stem diameter $(\mathrm{mm})$ using a caliper; e) number of leaves per plant; (f) fresh weight (kg); g) incidence of root gall using a grading scale ( 0 to 5 ), where 0 represents less than or equal to 5 galls; 1 represents more than 5 but less than or equal to 20 galls; 2 , greater than 20 but less than or equal to 40 galls; 3 , greater than 40 but less than or equal to $60 ; 4$, greater than 60 but less than or equal to 80 and $5=$ more than 80 galls (Gomes,1999).

\section{Statistical analysis}

The data were submitted to an analysis of variance (ANOVA) F test $(p=0.05)$ and the means were compared by the ScottKnott test $(p=0.05)$. Then, multivariate analyses were carried out to determinate genetic dissimilarity among the genotypes. This yielded a dissimilarity matrix based on a generalized distance of Mahalanobis $\left(\mathrm{D}_{\mathrm{ii}}^{2}\right)$.

Genetic divergence was represented by a dendrogram, which was obtained via the Unweighted Pair-Group Method Using Arithmetic Averages (UPGMA) and the optimization method of Tocher. Grouping by the UPGMA method was validated using the co-phenotype correlation coefficient (CCC) (Mantel test, 1967). The relative contribution of quantitative characteristics was calculated according to Singh (1981).

The cut-points in the dendrogram were established by determining the locations of abrupt level changes (Sudréet al., 2005). All data were analyzed using Genes v. 2015.5.0 (Cruz, 2013).

\section{Conclusion}

We found genetic diversity among the crisphead lettuce genotypes evaluated in the present study. The genotypes 199\#1\#1-E, 199\#2\#1-E, 199\#2\#2-E, 199\#3\#1-E, 199\#6\#1-E and $75 \# 2 \# 2-E$ are noteworthy because of their strong agronomic potential, and because they are biofortified and have a similar reaction to the root-knot nematode (Meloidogynesp.) as that of the Grand Rapids cultivar, which is considered resistant.

\section{Acknowledgments}

We would like to thank The Federal University of Uberlandia for providing the infrastructure and resources needed for this study.

\section{References}

Aquino CF, Silva HP; Neves JMG, Aquino F (2017) Desempenho de cultivares de alface sob cultivo 
hidropônico nas condições do norte de Minas Gerais. Rev Bras Agric Irrigad. 11: 1382-1388.

Abcsem (2013) Associação Brasileira do Comércio de Sementes e Mudas. Available at: <www.abcsem.com.br/dadosdosegmento.php>. Access in: December 20, 2017.

Carvalho Filho JLS, Gomes LAA, Maluf WR, Oliveira RR, Costa DS, Ferreira S, Monteiro AB, Costa e Carvalho RR (2011a) Resistance to Meloidogyne incognita race 1 in the lettuce cultivars Grand Rapids and Salinas-88. Euphytica. 182: 199208.

Carvalho Filho JLS, Gomes LAA, Silva RR, Ferreira S, Costa RR, Maluf WR (2011b) Parâmetros populacionais e correlação entre características da resistência a nematoides de galhas em alface. Rev Bras Ciênc Agrár. 6: 46-51.

Chapman MS (2012). Vitamin A: history, current uses, and controversies. Semin Cutan Med Surg. 31:11-16.

Cruz CD (2013) Genes: a software package for analysis in experimental statistics and quantitative genetics. Acta Sci. 35: 271-276.

Cruz CD, Regazzi AJ, Carneiro PCS (2012). Modelos biométricos aplicados ao melhoramento genético, 3a ed, Editora UFV, Viçosa, Minas Gerais.

Dias DP, Marenco RA (2007) Fotossíntese e fotoinibição em mogno e acariquara em função da luminosidade e temperatura foliar. Pesq Agropec Bras. 42: 305-311.

Feltrim AL, Cecílio Filho AB, Rezende BLA, Branco RBF (2009) Produção de alface-crespa em solo e em hidroponia, no inverno e verão, em Jaboticabal-SP. Científica. 37: 9-15.

Ferreira S, Gomes LAA, Gasparino CF, Carvalho Filho JLS, Maluf WR (2013) Caracterização de famílias F2:3 de alface para resistência ao nematoide das galhas. Rev Agrogeoambiental. 5: 35-42.

Ferreira RLF, Souza RJ de, Carvalho JG de, Araújo Neto SE de, Mendonça V, Wadt PGS (2010) Avaliação de cultivares de alface adubadas com silicato de cálcio em casa-devegetação. Ciênc e Agrotec. 34: 1093-1101.

Ferreira S, Vieira VLF, Gomes LAA, Maluf WR, Carvalho Filho JLS de (2011) Identificação de linhagens avançadas de alface quanto à resistência a Meloidogyne javanica. Ciênc e Agrotec. 35: 270-277.

Gomes LAA (1999). Herança da resistência da alface (Lactuca sativa L.) cv. Grand Rapids ao nematoide de galhas Meloidogyne incognita (Kofoid \& White) Chitwood. Lavras: UFLA.

Heredia Zárate NA, Vieira MC, Helmich M, Heid DM, Menegati CT (2010) Produção agroeconômica de três variedades de alface: cultivo com e sem amontoa. Rev Ciênc Agron. 41: 646-653.

Hermes CC, Medeiros SLP, Manfron PA, Caron B, Pommer SF, Bianchi C (2001) Emissão de folhas de alface em função de soma térmica. Rev Bras Agrometeorologia. 9: 269-275.
Institute of Medicine (2001). Dietary Reference Intakes for Vitamin A, Vitamin K, Arsenic, Boron, Chromium, Copper, lodine, Iron, Manganese, Molybdenum, Nickel, Silicon, Vanadium, and Zinc. Institute of Medicine, Washington, D.C

Jenkins WR (1964) A rapid centrifugal - flotation technique for separating nematodes from soil. Plant Diasease Report. 48: 692.

Klooster WS, Cregg BM, Fernandez RT, Nzokou P (2012) Growth and physiology of deciduous shade trees in response to controlled-release fertilizer. Sci Hortic.135: 7179.

Mantel N (1967) The detection of disease clustering and a generalized regression approach. Cancer Res. 27: 209-220.

Nick C, Carvalho SP de, Jesus MAS, Custódio TN, Marim BG, Assis LHB de (2010) Divergência genética entre subamostras de mandioca. Bragantia.69: 289-298.

Nunes GHS, Melo DRM de, Dantas DJ, Aragão FAS de, Pereira EWL (2011) Divergência genética entre linhagens de melão do grupo Inodorus. Rev Ciênc Agron. 42: 448456.

Oliveira ACB de, Sediyama MAN, Pedrosa MW, Garcia NCP, Garcia SLR (2004) Divergência genética e descarte de variáveis em alface cultivada sob sistema hidropônico. Acta Sci Agron. 26: 211-217.

Penniston KL, Tanumihardjo, SA ( 2006). The acute and chronic toxic effects of vitamin A. Am J Clin Nutr. 83:191201.

Ribeiro AC, Guimarães PTC, Alvarez VH (1999) 5a Aproximação - Recomendações para o uso de corretivos e fertilizantes em Minas Gerais. Editora UFV, Viçosa, Minas Gerais.

Sala FC, Costa CP (2012) Retrospectiva e tendência da alfacicultura brasileira. Hort Bras. 30: 187-194.

Santos CL dos, Seabra Junior S, Lalla JG de, Theodoro VCA, Nespoli A (2009) Desempenho de cultivares de alface tipo crespa sob altas temperaturas em Cáceres-MT. Agrarian. 2: 87-98.

Singh D (1981) The relative importance of characters affecting genetic divergence. Ind J Genet Plant Breed. 41: 237-245.

Sousa CS de, Bonetti AM, Goulart Filho LR, Machado JRA, Londe LN, Baffi MA, Ramos RG, Vieira CV, Kerr WE (2007) Divergência genética entre genótipos de alface por meio de marcadores AFLP. Bragantia. 66: 11-16.

Sudré $C P$, Rodrigues R, Riva EM, Karasawa $M$, Amaral Júnior AT do (2005) Divergência genética entre acessos de pimenta e pimentão utilizando técnicas multivariadas. Hort Bras. 23: 22-27.

Suinaga FA, Boiteux LS, Cabral CS, Rodrigues CS (2013) Desempenho produtivo de cultivares de alface crespa. Brasília, Distrito Federal, 15 p. (Boletim de Pesquisa e Desenvolvimento / Embrapa Hortaliças. 89. 